\title{
Corrigendum \\ On the Real Part of an Entire Function, its Derivative and its Lower Order
}

Mh. f. Math. [70, 244-248 (1966)]

By

T. V. Lakshminarasimhan, Ramanujan Institute, Madras-5, India

(Received August 26, 1968)

Dr. S. JAENISCH drew my attention to a mistake in the proof of my result stated as Theorem $B$ in the paper referred to above. The mistake arises out of an erroneous lemma, stated as Lemma 2 in the paper, and its application in the course of the proof. Freed from this mistake, the proof of Theorem $B$ yields the following result.

Theorem $\mathrm{B}^{\prime}$. If $f(z)$ is an entire function of lower order $\lambda, 0 \leq \lambda \leq \infty$, then

$\lim _{r \rightarrow \infty} \inf \frac{\log \left(\max _{|z|=r}\left|\operatorname{Re} z f^{\prime}(z)\right| / \max _{|z|=r}|\operatorname{Re} f(z)|\right)}{\log r} \leq \lambda$.

Clearly the above inequality reduces to an equality when $\lambda=0$ and becomes trivial when $\lambda=\infty$.

The Proof is essentially as in page 247 and runs as follows after the correction of some minor errors.

In Rajagopal's lemma, quoted by me as Lemma 1, corresponding to each $|z|=r$ belonging to a sequence of ordinary values of $r$ of index $11 / 12$ tending steadily to $\infty$, there is a $\zeta$ such that $|\zeta|=r$ and

But

$$
\left|\operatorname{Re} \zeta f^{\prime}(\zeta)\right| \sim v(r) \max _{|z|=r}|\operatorname{Re} f(z)| .
$$

$$
\begin{gathered}
\left|\operatorname{Re} \zeta f^{\prime}(\zeta)\right| \leq \max _{|z|=r}\left|\operatorname{Re} z f^{\prime}(z)\right| \\
\leq r M^{\prime}(r) \sim v(r) M(r) \sim v(r) \max _{|z|=r}|\operatorname{Re} f(z)|
\end{gathered}
$$


by a result of Valiron's cited by Rajagopal and his own arguments. Hence

$$
\max _{|z|=r}\left|\operatorname{Re} z f^{\prime}(z)\right| \sim v(r) \max _{|z|=s}|\operatorname{Re} f(z)| .
$$

Now, choosing the sequence of ordinary values of $r$ tending to $\infty$ to be $\left\{e_{n}\right\}$, as defined in the proof of my Theorem $\mathrm{B}$ on page 247 , we get

$$
\begin{aligned}
\max _{|z|=e_{n}}\left|\operatorname{Rez} f^{\prime}(z)\right| & \sim v\left(e_{n}\right) \max _{|z|=e_{n}}|\operatorname{Ref}(z)| \\
& <2 v\left(e_{n}\right) \max _{|z|=e_{n}}|\operatorname{Re} f(z)|\left(n>n_{0}\right) \\
& <2 e_{n}^{\lambda+\varepsilon} \max _{|z|=e_{n}}|\operatorname{Ref}(z)|\left(n>n_{1}>n_{0}\right) .
\end{aligned}
$$

From this the conclusion of Theorem B' follows at once.

The erroneous result stated by me as Lemma 2, though not used in the above proof, may, be corrected as follows.

Lemma $2^{\prime}$. For an entire function $f(z)=u(x, y)+i v(x, y)$, where $z=x+i y$, the condition $|f| \leq C u(C=a$ const $)$ implies that $f=a$ const.

Proof. We have $v \leq \sqrt{\left(u^{2}+v^{2}\right)}=|f|<C u$, so that $v-C u<0$ and

$$
F(z)=\exp \left(\frac{1}{i}-C\right)(u+i v)
$$

satisfies the condition

$$
|F|<\exp (v-C u)<1 .
$$

Thus, by Liouville's theorem, $F$ reduces to a constant and therefore also $f$.

I thank Prof. C. T. RAJAGOPAL who has helped me clarify some essential points and drawn my attention to the following misprints in his paper (Monatsh. Math. 66, S. 339-345) cited by me.

Page 340, line 9. For $\operatorname{Re} h^{\prime \prime}$ read $h^{\prime \prime}$, adding $h^{\prime \prime}=\operatorname{Re} h$ at end.

Page 342, line 7. For $\nu(r)<r^{o-\varepsilon} \operatorname{read} \nu(r)>r^{o-\varepsilon}$.

Added in proof: The present note is a sequel to a previous note entitled "Corrigendum and Addendum to my paper On the real part of an entire function." 